\title{
Side Population Cell Level in Human Breast Cancer and Factors Related to Disease-free Survival
}

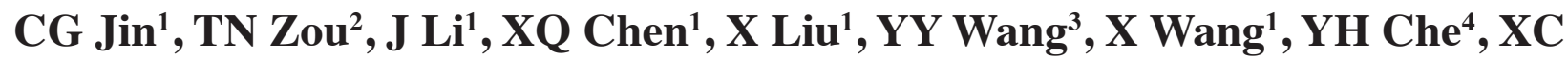 \\ Wang ${ }^{1}$, Hutcha Sriplung ${ }^{5 *}$
}

\begin{abstract}
Side population (SP) cells have stem cell-like properties with a capacity for self-renewal and are resistant to chemotherapy and radiotherapy. Therefore the presence of SP cells in human breast cancer probably has prognostic value. Objective: To investigate the characteristics of SP cells and identify the relationship between the SP cells levels and clinico-pathological parameters of the breast tumor and disease-free survival (DFS) in breast cancer patients. Materials and Methods: A total of 122 eligible breast cancer patients were consecutively recruited from January 1, 2006 to December 31, 2007 at Yunnan Tumor Hospital. All eligible subjects received conventional treatment and were followed up for seven years. Predictors of recurrence and/or metastasis and DFS were analyzed using Cox regression analysis. Human breast cancer cells were also obtained from fresh human breast cancer tissue and cultured by the nucleic acid dye Hoechst33342 with Verapami. Flow cytometry (FCM) was employed to isolate the cells of SP and non-SP types. Results: In this study, SP cells were identified using flow cytometric analysis with Hoechst 33342 dye efflux. Adjusted for age, tumor size, lymph nodal status, histological grade, the Cox model showed a higher risk of recurrence and/or metastasis positively associated with the SP cell level $(1.75,1.02-2.98)$, as well as with axillary lymph node metastasis $(2.99,1.76-5.09)$, pathology invasiveness type $(1.7,1.14-2.55)$, and tumor volume doubling time (TVDT) $(1.54,1.01-2.36)$. Conclusions: The SP cell level is independently associated with tumor progression and clinical outcome after controlling for other pathological factors. The axillary lymph node status, TVDT and the status of non-invasive or invasive tumor independently predict the prognosis of breast cancer.
\end{abstract}

Keywords: Breast cancer - side population cell - cancer stem cell - prognosis

Asian Pac J Cancer Prev, 16 (3), 991-996

\section{Introduction}

While overall cancer incidence has been increasing in China during the recent years, breast cancer has become the top one in females. The relative survival rate in Chinese patients with breast cancer was $78.7 \%$ in the period 1992 1995 (Sankaranarayanan et al., 2011). The majority of breast cancer deaths occur as a result of recurrence or metastasis of the disease rather than from the effects of the primary tumor and cancer stem cell (CSC) is an important character in metastatic behavior (Croker et al., 2009).

According to the theory of stem cell, the most important and useful property of stem cells is that of self-renewal. This is a common property of both stem cells and cancer cells since tumours often originate from the transformation of normal stem cells, thus, similar signaling pathways may regulate self-renewal in both stem cells and cancer cells. Most cancer cells are considered to be differentiated from CSC - rare cells with self-renewal ability that drive tumorigenesis (Reya et al., 2001). Studies have identified tumorigenic cells with stem/progenitor cell properties from human breast cancers (Ponti et al., 2005). In identification and isolation of CSCs, several approaches have been proposed. In breast cancers, cell surface markers, such as CD24 and CD44, have been proved to be useful for the isolation of subsets enriched for breast CSCs (Al-Hajj et al., 2003; Visvader, 2008).

In 1996, Goodell MA, et al. discovered and isolated a special population of cells while working with staining of murine bone marrow cells with the vital dye, Hoechst 33342, and named them as side population (SP) cells (Goodell et al., 1996). These cells shared characteristics of CSCs and were enriched in tumor initiating capacity and expressed stem-like genes (Zhang et al., 2012). SP cells have later been identified in both mouse and human mammary gland tissue (Alvi et al., 2003; Clayton et al.,

${ }^{1}$ Cancer Research Institute, ${ }^{2}$ Breast Cancer Department, Yunnan Cancer Hospital, The 3rd Affiliated Hospital of Kunming Medical University, ${ }^{4}$ The First Peoples Hospital of Kunming City, Yunnan Province, ${ }^{3}$ Epidemiology and Biostatistics Department, School of Public Health, Kunming Medical University, China, ${ }^{5}$ Epidemiology Unit, Faculty of Medicine, Prince of Songkla University, Songkhla, Thailand *For correspondence: hutcha.s@gmail.com 
2004). The cells have been identified in a variety of normal tissues and cancers and have shown to exhibit stem celllike properties in capable of self-renewal (Wu and Alman, 2008; Nakanishi et al., 2010). SP cells have also been found to be chemoresistant (Song J et al., 2010; Yang M et al., 2010), radioresistant (Woodward et al., 2007) and have increased invasive potential exhibited in vitro (Fuchs et al., 2009) compared to nonside population (NSP) cells. In vivo studies have also demonstrated that in most cancer cell lines SP are more tumourigenic (Yin et al., 2008; Chen et al., 2009; Song et al., 2010) and have greater metastatic potential (Chen et al., 2009; Nishii et al., 2009) than their NSP counterparts (Mitsutake et al., 2007).

There exist many factors having the potential to become relevant predictive factors in the past year (Cianfrocca and Goldstein, 2004). Prognostic and predictive factors for breast cancer include classical clincopathological features derived from breast cancer samples including stage, tumor size, histological subtype and grade, lymph node metastases (Rosa Mendoza ES et al., 2013; Nogami T et al., 2014 ), type of tumor, lymphatic and vascular invasion (Jatoi et al., 1999 ), and hormone receptor status (Weigel, 2010).

The early detection of cancer and improvements of cancer treatments provide better progress for cancer, however, the ability to prolong cancer survival is still limited. It is important to evaluate the potential role of these SP cells in tumor metastasis and recurrence of patients with breast cancer. This study aimed to investigate the proportion of SP cells, the factors associated with their presence and to identify the effect of SP cells in the human breast cancer cell on prognosis of patients with breast cancer

\section{Materials and Methods}

\section{Study subjects}

Breast cancer patients who were diagnosed according to the National Comprehensive Cancer Network (NCCN) Clinical Practice Guidelines (Sun et al., 2011). Those complicated with chronic wasting and infectious diseases, or having multiple primary malignancies were excluded. Based on the criteria above, breast cancer patients diagnosed from January 1, 2006 to December 31, 2007 and in a maximum 7-year follow up period that observed both the occurred recurrence or metastasis and also those without these outcomes after completed conventional treatment were consecutively recruited in this study at Yunnan Tumor Hospital. The human breast cancer cells were obtained from fresh tissue of the cancer patients. The cells were cultured by the nucleic acid dye Hoechst33342 added with Verapami. Flow cytometry (FCM) was employed to isolate the cells of SP.

The protocol of this study was approved by the Ethical Committee of Yunnan Tumor Hospital. All eligible participants were informed of the study protocol and informed consent was signed before the patients accepted admission into the study.

\section{Cell culture and flow cytometry analysis}

Cell culture for each patient was performed at $10 \%$
FBS RPMI-1640 (Gibco) medium in a humidified $37^{\circ} \mathrm{C}, 5 \% \mathrm{CO}_{2}$ in Forma311 carbon dioxide incubator (Thermo Fisher Scientific, USA). Cells were selected in the logarithmic growth phase, removed from the culture dish with trypsin and EDTA, pelleted by centrifugation, washed with PBS. The cell suspension diluted to 106/ ml with $10 \%$ FBS RPMI-1640 solution. The cells were then labeled with Hoechst 33342 (Sigma-Aldrich) at a concentration of $5 \mu \mathrm{g} / \mathrm{ml}$. The labeled cells were incubated for 120 minutes at $37^{\circ} \mathrm{C}$, either alone or with $100 \mu$ l verapamil hydrochloride (Sigma-Aldrich) and were swirled every 15 minutes. Two hours later, $4^{\circ} \mathrm{C}$ PBS was used to terminate labeled process. After centrifugation with desk centrifuge (Heraeus, Germany), the cells were washed with $2 \%$ fetal bovine serum (FBS; Gibco) PBS and maintained at $4{ }^{\circ} \mathrm{C} 2 \%$ FBS PBS and were processed for flow cytometry analysis. Cells were counterstained with $2 \mu \mathrm{g} / \mathrm{ml}$ propidium iodide (PI; Sigma-Aldrich) to identify dead cells. Then 1 x $10^{6}$ viable cells were analyzed and sorted by an Epics Altra flow cytometry (Beckmancoulter, USA). The Hoechst dye was excited at $407 \mathrm{~nm}$ UV (blue, 450/40; red, 695/40). PI was excited at $488 \mathrm{~nm}$ blue (red, 575/26).

\section{Clinical data collection}

All recruited subjects were recorded on basic demographics such as age at diagnosis, basic clinical and pathological information such as tumor size, clinical stage and pathological findings. Records for this information were independent with cell culture and cytometry results. Both data were later merged by patient identification number. Recurrence or metastasis status as the outcome of this follow up study was recorded by schedule revisiting of patients.

\section{Data analysis}

Descriptive statistics methods were adopted to present the distribution of data. T test was utilized to compare the differences of distribution of SP cell with age group at the cut point of 60 years old and 6 tumor characteristics. Kaplan-Meier was used to descript the distributions of DFS and log rank test was adopted to detect difference of median survival time between groups. Cox regression models were employed to explore the risk factors of disease free survival. Modeling used the manual backward exclusion method, sequentially removing variables not contributing significantly to the fit of the model based on the likelihood ratio of successive models. SP status, clinical and pathological information were all included in the first full model. All the tests were as two-sided and $\mathrm{P}$ value of less than 0.05 was generally considered as statistical significance.

\section{Results}

A total of 122 patients had recurrence or metastasis and 524 patients had no evidence of the outcome of interest. Among these 122 patients, the range of proportion of SP cell was $1.1 \%$ to $6.2 \%$ with the mean of proportion of $3.2 \%$ and standard deviation of $1.2 \%$. Table 1 shows the percentage of SP cell grouped by age 
Side Population Cells in Breast Cancer and Disease-free Survival

and tumor characteristics. Distribution of SP cell differed significantly across 6 tumor characteristics. Higher SP cell was common in the more serious status of breast cancer than in the lighter illness status.

Disease-free survival (DFS) was compared among

Table 1. Distribution of SP Cells Level In Demographic And Tumor Characteristics For Patients With Breast Cancer

\begin{tabular}{|c|c|c|c|}
\hline Factors & No. & $\begin{array}{l}\text { SP cells level } \\
(\text { mean } \pm \text { sd })\end{array}$ & $\mathrm{p}$ value \\
\hline \multicolumn{4}{|l|}{ Age } \\
\hline$<60$ year & 81 & $3.28 \pm 1.25$ & \\
\hline$\geq 60$ year & 41 & $3.20 \pm 1.14$ & 0.695 \\
\hline \multicolumn{4}{|l|}{ Tumor size } \\
\hline$<2 \mathrm{~cm}$ & 73 & $3.00 \pm 1.18$ & \\
\hline$\geq 2 \mathrm{~cm}$ & 49 & $3.62 \pm 1.17$ & 0.005 \\
\hline \multicolumn{4}{|l|}{ ALNM } \\
\hline$<2$ nodes & 74 & $2.73 \pm 1.03$ & \\
\hline$\geq 2$ nodes & 48 & $4.04 \pm 1.02$ & 0.000 \\
\hline \multicolumn{4}{|c|}{ Pathology invasiveness } \\
\hline non-invasive & 69 & $3.04 \pm 1.16$ & \\
\hline invasive & 53 & $3.52 \pm 1.22$ & 0.028 \\
\hline \multicolumn{4}{|l|}{ Clinical stage } \\
\hline I / II & 61 & $2.81 \pm 1.08$ & \\
\hline III & 61 & $3.69 \pm 1.17$ & 0.000 \\
\hline \multicolumn{4}{|l|}{ Differentiated degree } \\
\hline High & 56 & $2.94 \pm 1.11$ & \\
\hline Low & 66 & $3.51 \pm 1.23$ & 0.009 \\
\hline \multicolumn{4}{|l|}{ TVDT } \\
\hline Fast growing & 59 & $2.80 \pm 1.16$ & \\
\hline Slow growing & 63 & $3.67 \pm 1.10$ & 0.000 \\
\hline
\end{tabular}

patients' age group less that 60 or equal or above, tumor characteristics and SP cell levels of less than or equal and above $3.6 \%$. Statistics significant difference was determined by the tumor characteristics as well as SP cell levels (Table 2). The significant difference in cumulative survival was detected by $\log$ rank test $(\mathrm{p}<0.001)$ for the two categories of SP cell levels among breast cancer patients (Figure 1).

Four out of all 8 variables were identified as significant independent risk factors for disease-free survival in multivariate Cox regression model. Those factors were axillary lymph node metastasis (ALNM), invasiveness of the tumor, tumor volume doubling time (TVDT) and SP cell level (Table 3).

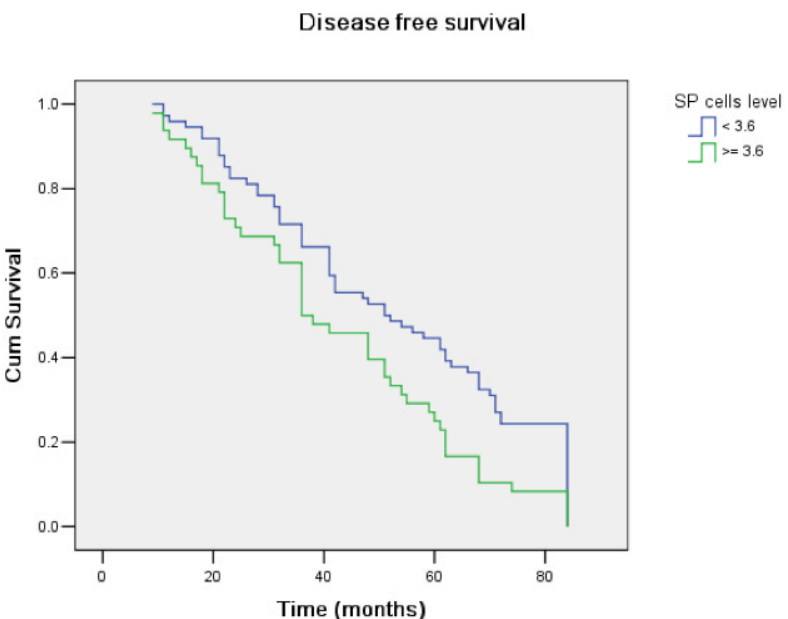

Figure 1. The Disease-free Survival of Breast Cancer Patients with Different Levels of SP Cells

Table 2. Disease-free Survival Probability with Subsequent Recurrence and Metastasis for Patients with Breast Cancer

\begin{tabular}{|c|c|c|c|c|c|c|c|}
\hline \multirow[t]{2}{*}{ Factors } & \multirow[t]{2}{*}{ No. } & \multicolumn{4}{|c|}{ Disease-free survival } & \multirow[t]{2}{*}{$95 \% \mathrm{CI}^{*}$} & \multirow[t]{2}{*}{$\mathrm{p}$ value } \\
\hline & & $12 \mathrm{mo.}$ & $36 \mathrm{mo.}$ & $60 \mathrm{mo.}$ & an (months) & & \\
\hline \multicolumn{8}{|l|}{ Age } \\
\hline$<60$ years & 81 & 0.951 & 0.605 & 0.346 & 47.0 & $38.2-55.8$ & \\
\hline$\geq 60$ years & 41 & 0.927 & 0.585 & 0.415 & 48.0 & $30.4-65.6$ & 0.680 \\
\hline \multicolumn{8}{|l|}{ Tumor size } \\
\hline$<2 \mathrm{~cm}$ & 73 & 0.945 & 0.671 & 0.452 & 54.0 & $43.3-64.6$ & \\
\hline$\geq 2 \mathrm{~cm}$ & 49 & 0.939 & 0.490 & 0.245 & 36.0 & $29.1-42.8$ & 0.005 \\
\hline \multicolumn{8}{|l|}{ ALNM } \\
\hline$<2$ nodes & 74 & 0.986 & 0.811 & 0.527 & 61.0 & $55.9-66.0$ & \\
\hline$\geq 2$ nodes & 48 & 0.854 & 0.271 & 0.125 & 32.0 & $24.3-39.7$ & 0.000 \\
\hline \multicolumn{8}{|c|}{ Pathology invasiveness } \\
\hline non-invasive & 69 & 0.942 & 0.638 & 0.435 & 52.0 & $39.2-64.8$ & \\
\hline invasive & 53 & 0.943 & 0.547 & 0.264 & 41.0 & $35.2-46.8$ & 0.001 \\
\hline \multicolumn{8}{|l|}{ Clinical stage } \\
\hline I / II & 61 & 0.984 & 0.754 & 0.459 & 58.0 & $48.4-67.6$ & \\
\hline III & 61 & 0.885 & 0.443 & 0.262 & 36.0 & $32.2-39.8$ & 0.000 \\
\hline \multicolumn{8}{|c|}{ Differentiated degree } \\
\hline high & 56 & 0.929 & 0.679 & 0.464 & 59.0 & $50.0-68.0$ & \\
\hline low & 66 & 0.955 & 0.530 & 0.258 & 41.0 & $32.5-49.5$ & 0.002 \\
\hline \multicolumn{8}{|l|}{ TVDT } \\
\hline Fast growing & 59 & 0.949 & 0.712 & 0.475 & 61.0 & $50.3-71.7$ & \\
\hline Slow growing & 63 & 0.937 & 0.492 & 0.238 & 36.0 & $29.6-42.4$ & 0.001 \\
\hline \multicolumn{8}{|l|}{ SP cells level } \\
\hline$<3.6 \%$ & 74 & 0.959 & 0.662 & 0.419 & 51.0 & $34.1-67.9$ & \\
\hline$\geq 3.6 \%$ & 48 & 0.917 & 0.500 & 0.250 & 36.0 & $26.1-45.9$ & 0.010 \\
\hline
\end{tabular}


Table 3. Predictors in the Final Cox Regression Model for Disease-free Survival in Patients with Breast Cancer and Sp Cell Level and Tumor Characters

\begin{tabular}{|c|c|c|}
\hline & $\mathrm{HR}(95 \% \mathrm{CI})$ & $* \mathrm{p}$ value \\
\hline \multicolumn{3}{|c|}{ Axillary lymph node metastasis } \\
\hline$<2$ & Ref. & \\
\hline$\geq 2$ & $2.99(1.76-5.09)$ & 0.000 \\
\hline \multicolumn{3}{|c|}{ Pathology invasiveness } \\
\hline non-invasive & Ref. & \\
\hline invasive & $1.7(1.14-2.55)$ & 0.010 \\
\hline \multicolumn{3}{|c|}{ Tumor volume doubling time } \\
\hline fast & Ref. & \\
\hline slow & $1.54(1.01-2.36)$ & 0.044 \\
\hline \multicolumn{3}{|l|}{ SP cell level } \\
\hline$<3.6 \%$ & Ref. & \\
\hline$\geq 3.6 \%$ & $1.75(1.02-2.98)$ & 0.041 \\
\hline
\end{tabular}

\section{Discussion}

In this study, the SP cells were identified by a flow cytometric analysis using Hoechst 33342 dye efflux and the mean of SP cell proportion in breast cancer tissue was $3.25 \pm 1.21 \%$. Previous studies have reported the percentages of SP cells to range from $0.2 \%$ to $7.5 \%$ (Patrawala et al., 2005; Engelmann et al., 2008; Han, 2009). Such the variation could be determined by the difference in machines and reagents used in different studies. In order to better understand the relationship between the percentage of SP cells and prognosis of breast cancer patients, since the standard had not been set, we attempted to select the cutoff point at $3.6 \%$ in this study which based on the mean of SP cells percentage. Under the control of age and other tumor pathological characteristics, the SP cell level equal to or more than $3.6 \%$ was an independent risk factor of DFS among breast cancer patients. In the univariate analysis of this study, SP cell level and the pathological factors named tumor size, ALNM, invasiveness of the tumor, stage, degree of differentiation and TVDT were statistically significant related to DFS. However, only SP cells level, invasiveness, ALNM and TVDT were independent factors which statistically significant correlated with prognoses after all variables were added for adjustment of inter-dependency in the Cox model.

The study clearly showed a higher hazard ratio of 1.75 (Table 3) among those with higher level of SP cells. Such the phenomenon could be in part explained by the nature of CSC in which SP cells are among them. CSCs are considered to be responsible for tumor initiation and progression. The residual CSC may involved in the process of tumor metastasis and tumor progression which is a result of the metastatic spread of these cells (Campbell, 2007). Although chemotherapy and radiotherapy would have killed most cells and cell lines in a tumour, it is believed to leave tumour stem cells behind as they are resistant to the treatment modalities. While those treatments could reduce large amount of cancer cells and cause observable tumor shrinkage, the effect on CSC could be minimal (Mimeault et al., 2007; Raaijmakers, 2007; Sakariassen et al., 2007; Zeppernick et al., 2008). CSC might also be one of important mechanisms of multidrug resistance that leads to tumor recurrence after chemotherapy. The SP cells possessing stem cell-like properties have been found to be associated with a poorer prognosis (Van den Broeck et al., 2013). Most of patients with cancer with or without cell killing therapeutic methods could progress and/or develop metastases. Although there is no literature investigating the SP cells and metastatic probability in breast cancer, studies in other cancers have elaborated an important role of SP cells in metastasis of cancer (Kato et al., 2007; Nishii et al., 2009). The study of Kato and co-workers on cultured SP and NSP cells from the human endometrial cancer cell disclosed that tumours derived from SP cells showed the characteristics of progenitor cells. The progenitor cell potential of SP cells showed long-term repopulation properties ( $>24$ weeks), and cultured SP cells produced gland (CD9+)- and stroma (CD13+)-like cells. In contrast, NSP cells became senescent within 1-3 months (Kato et al., 2007). SP isolated from the pancreatic cancer cell line PANC-1 have been shown to have an increased invasive potential in vitro and increased metastatic potential in vivo when compared to NSP cells using a murine liver metastasis model (Kabashima et al., 2009). Nishii and colleagues in 2009 have shown SP cells from gastric cancer increased a high adhesion ability to peritoneum related to the expression of several adhesion molecules which resulted in SP cells having a greater potential for peritoneal metastases (Nishii et al., 2009). In this study we divided SP cells size into two levels based on the mean size instead of NSP cells. The results found that the patients' SP cells levels less than 3.6\% has longer median DFS than those equal to or more than $3.6 \%$ SP cells level which means it had better progress. It is probably be explained by above research results, in NSP cells had less metastasis. Thus the finding from this study is closed to those studies results.

The significantly higher hazard ratio for metastasis and recurrence in the group of patients with SP cells level equal and more than $3.6 \%$ than the group with less than $3.6 \%$ SP cells (Table 3) is consisted with the results in previous studies which illustrated SP cells being an important element for breast cancer prognosis. The breast cancer SP cells in vivo have been shown to be more tumourigenic than NSP cells when tumor cell cultures in immunodeficient mice (Patrawala et al., 2005; Steiniger et al., 2008; Yin et al., 2008). In Zhou et al. (2008) study, the sphere cells cultured from SP cells of breast cancer cell MCF7 were enriched in CSC surface markers CD44+CD24- and had higher tumorigenicity. It implied the sphere cells enrich breast cancer stem/ progenitor cells and preferentially inhibited by NF- $x \beta$ pathway inhibitors compared to their non-stem cell counterparts. Studies have demonstrated that MCF7 SP in the mammary gland are more clinically relevant doses of radiation resistant (Woodward et al., 2007) and clinical resistance to chemotherapy (Steiniger et al., 2008; Yin et al., 2008) than the NSP cell population. These properties would suggest that SP cells may also play a key role in breast cancer progression.

In addition to the levels of SP cells, Table 3 shows other 
important determinants of DFS being ipsilateral axillary lymph node metastasis (ALNM), tumor invasiveness, and tumor volume doubling time (TVDT). Axillary lymph node status is the most important prognostic variable in the management of patients with primary breast cancer. A study (Jatoi et al., 1999) illustrated that DFS after relapse was poorer in node-positive cases compared with node-negative cases. The hazard ratio for patients with one to three node involvement compared with none was $1.2(95 \% \mathrm{CI}=0.8-1.9)$ and it was $2.5(95 \% \mathrm{CI}=1.8-3.4)$ among four or more involved nodes, respectively. Other studies reported the significant higher HR of DFS among patients with than those without axillary lymph node metastasis (ALNM) (Rosa Mendoza et al., 2013; Nogami et al., 2014 ).

As shown in Table 3 that TVDT was an independent variable in the final Cox model in predicting DFS. TVDT reflects the natural tumor growth rate and is an indicator of the biological malignant potential of a tumor. The evaluation of TVDT is able to provide a parameter as progress of cancer. The study of Kusama et al. (1972) in 34 cases with breast cancer illustrated that the growth of secondary tumor was related to that of the primary site.

Invasive status of the tumor was found to be one of the predictors of DFS in our analysis. Tumor invasiveness would determine treatment choices and the response to the treatment received. Non-invasive breast cancers are limited to the basement membrane of the milk ducts or lobules (the round sacs in the breast that produce milk) while invasive ones break such the barrier into the surrounding breast tissue and could have been spreading to the lymphatic system and then to other parts of the body. Therefore, invasive carcinomas have poorer prognosis and survival than non-invasive tumors.

In conclusion, SP cells level has been demonstrated to have independent association with tumor progression and clinical outcome after controlling for other clinical and pathological factors. Axillary lymph node status, TVDT and invasiveness of cancer were also identified as independently predictors for the prognosis of breast cancer.

\section{References}

Al-Hajj M, Wicha MS, Benito-Hernandez A, et al (2003). Prospective identification of tumorigenic breast cancer cells. Proc Natl Acad Sci USA, 100, 3983-8.

Alvi AJ, Clayton H, Joshi C, et al (2003). Functional and molecular characterisation of mammary side population cells. Breast Cancer Res, 5, 1-8.

Campbell LL and Polyak K (2007). Breast tumor heterogeneity: cancer stem cells or clonal evolution? Cell Cycle, 6, 2332-8.

Chen CY, Chiou SH, Huang CY, et al (2009). Distinct population of highly malignant cells in a head and neck squamous cell carcinoma cell line established by xenograft model. $J$ Biomed Sci, 16, 100.

Cianfrocca M and Goldstein LJ (2004). Prognostic and predictive factors in early-stage breast cancer. Oncologist, 9, 606-16.

Clayton H, Titley I and Vivanco M (2004). Growth and differentiation of progenitor/stem cells derived from the human mammary gland. Exp Cell Res, 297, 444-60.

Croker AK, Goodale D, Chu J, et al (2009). High aldehyde dehydrogenase and expression of cancer stem cell markers selects for breast cancer cells with enhanced malignant and metastatic ability. J Cell Mol Med, 13, 2236-52.

Engelmann K, Shen H and Finn OJ (2008). MCF7 side population cells with characteristics of cancer stem/ progenitor cells express the tumor antigen MUC1. Cancer Res, 68, 2419-26.

Fuchs D, Heinold A, Opelz G, et al (2009). Salinomycin induces apoptosis and overcomes apoptosis resistance in human cancer cells. Biochem Biophys Res Commun, 390, 743-9.

Goodell MA, Brose K, Paradis G, et al (1996). Isolation and functional properties of murine hematopoietic stem cells that are replicating in vivo. J Exp Med, 183, 1797-806.

Han JS and Crowe DL (2009). Tumor initiating cancer stem cells from human breast cancer cell lines. Int J Oncol, 34, 1449-53.

Jatoi I, Hilsenbeck SG, Clark GM, et al (1999). Significance of axillary lymph node metastasis in primary breast cancer. $J$ Clin Oncol, 17, 2334-40.

Kabashima A, Higuchi H, Takaishi H, et al (2009). Side population of pancreatic cancer cells predominates in TGF- $\beta$-mediated epithelial to mesenchymal transition and invasion. Int J Cancer, 124, 2771-9.

Kato K, Yoshimoto M, Kato K, et al (2007). Characterization of side-population cells in human normal endometrium. Hum Reprod, 22, 1214-23.

Kusama S, Spratt JS, Donegan WL, et al (1972). The gross rates of growth of human mammary carcinoma. Cancer, 30, 594-9.

Mimeault M, Hauke R, Mehta PP, et al (2007). Recent advances in cancerstem/progenitor cell research: therapeutic implications for overcomingresistance to the most aggressive cancers. J Cell Mol Med, 11, 981-1011.

Mitsutake N, Iwao A, Nagai K, et al (2007). Characterization of side population in thyroid cancer cell lines: Cancer stem-like cells are enriched partly but not exclusively. Endocrinology, 148, 1797-803.

Nakanishi T, Chumsri S, Khakpour N, et al (2010). Sidepopulation cells in luminal-type breast cancer have tumour-initiating cell properties, and are regulated by HER2 expression and signalling. Br J Cancer, 102, 815-26.

Nishii T, Yashiro M, Shinto O, et al (2009). Cancer stem cell-like SP cells have a high adhesion ability to the peritoneum in gastric carcinoma. Cancer Sci, 100, 1397-402.

Nogami T, Shien T, Tanaka T, et al (2014). Expression of ALDH1 in axillary lymph node metastases is a prognostic factor of poor clinical outcome in breast cancer patients with 1-3 lymph node metastases. Breast Cancer, 21, 58-65.

Patrawala, L., Calhoun T, R. Schneider-Broussard, et al (2005). Side population is enriched in tumorigenic, stem-like cancer cells, whereas ABCG2+ and ABCG2- cancer cells are similarly tumorigenic. Cancer Res, 65, 6207-19.

Ponti D, Costa A, Zaffaroni N, et al (2005). Isolation and in vitro propagation of tumorigenic breast cancer cells with stem/progenitor cell properties. Cancer Res, 65, 5506-11.

Raaijmakers MH (2007). ATP-binding-cassette transporters in hematopoieticstem cells and their utility as therapeutical targets in acute and chronicmyeloid leukemia. Leukemia, 21, 2094-02.

Reya T, Morrison SJ, Clarke MF, et al (2001). Stem cells, cancer, and cancer stem cells. Nature, 414, 105-11.

Rosa Mendoza ES, Moreno E and Caguioa PB (2013). Predictors of early distant metastasis in women with breast cancer. $J$ Cancer Res Clin Oncol, 139, 645-52.

Sakariassen PO, Immervoll H, Chekenya M (2007). Cancer stem cells as mediators of treatment resistance in brain tumors : status and controversies. Neoplasia, 9, 882-92. 
Sankaranarayanan R, Swaminathan R, Lucas E (2011). Cancer survival in Africa, Asia, the Caribbean and Central America (SurvCan). IARC scientific publications volume 162, ISBN 978-92-832-2162-3, Lyon, International Agency for Research on Cancer.

Song J, Chang I, Chen Z, et al (2010). Characterization of side populations in hnscc: highly invasive, chemoresistant and abnormal wnt signaling. PLoS One, 5, 11456.

Steiniger SC, Coppinger JA, Kruger JA, et al (2008). Quantitative mass spectrometry identifies drug targets in cancer stem cell containing side population. Stem Cells, 26, 3037-46.

Sun Y, Liao M, Cheng G (2011). NCCN non-small cell lung cancer clinical practice guideline: Chinese Edition Version 2011. Beijing.

Van den Broeck A, Vankelecom H, Van DelmW, et al (2013). Human pancreatic cancer contains a side population expressing cancer stem cell-associated and prognostic genes. PloS One, 8, 73968.

Visvader JE, Lindeman GJ (2008). Cancer stem cells in solid tumours: accumulating evidence and unresolved questions. Nat Rev Cancer, 8, 755-68.

Weigel MT, Dowsett M (2010). Current and emerging biomarkers in breast cancer: prognosis and prediction. Endocr Relat Cancer, 17, 245-62.

Woodward WA, Chen MS, Behbod F, et al (2007). WNT/betacatenin mediates radiation resistance of mouse mammary progenitor cells. Proc Natl Acad Sci USA, 104, 618-23.

Wu C and Alman BA. (2008) Side population cells in human cancers. Cancer Lett, 268, 1-9.

Yang M, Zhang R, Yan M, et al (2010). Detection and characterization of side population in Ewing's sarcoma SK-ES-1 cells in vitro. Biochem Biophys Res Commun, 391, 1062-6.

Yin L, Castagnino P, Assoian RK (2008). ABCG2 Expression and side population abundance regulated by a transforming growth factor $\{$ beta\}-directed epithelial-mesenchymal transition. Cancer Res, 68, 800-7.

Zeppernick F, Ahmadi R, Campos B, et al (2008). Stem cell markercd133 affects clinical outcome in glioma patients. Clin Cancer Res, 14, 123-9.

Zhang AM, Fan Y, Yao Q, Ma H, et al (2012). Identification of a cancer stem-like population in the Lewis lung cancer cell line. Asian Pac J Cancer Prev, 13, 761-6.

Zhou J, Zhang H, Gu P, et al (2008). Cancer stem/progenitor cell active compound 8-quinolinol in combination with paclitaxel achieves an improved cure of breast cancer in the mouse model. Breast Cancer Res Treat, 115, 269-77. 